\title{
Measurement of $\alpha$-particle quenching in LAB based scintillator in independent small-scale experiments
}

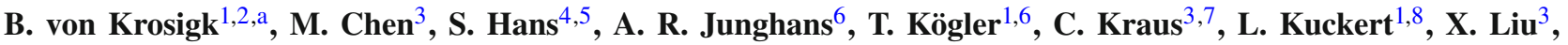 \\ R. Nolte ${ }^{9}$, H. M. O'Keeffe ${ }^{3,10}$, H. Wan Chan Tseung ${ }^{11,12}$, J. R. Wilson ${ }^{13}$, A. Wright ${ }^{3}$, M. Yeh ${ }^{4}$, K. Zuber ${ }^{1}$ \\ ${ }^{1}$ Institut für Kern- und Teilchenphysik, Technische Universität Dresden, 01069 Dresden, Germany \\ 2 Department of Physics and Astronomy, University of British Columbia, Vancouver, BC V6T 1Z1, Canada \\ ${ }^{3}$ Department of Physics, Engineering Physics and Astronomy, Queen's University, Kingston, ON K7L 3N6, Canada \\ ${ }^{4}$ Brookhaven National Laboratory, Upton, NY 11973, USA \\ ${ }^{5}$ Bronx Community College, Bronx, NY 10453, USA \\ ${ }^{6}$ Helmholtz-Zentrum Dresden-Rossendorf, 01314 Dresden, Germany \\ ${ }^{7}$ Laurentian University, 935 Ramsey Lake Road, Sudbury, ON P3E 2C6, Canada \\ ${ }^{8}$ Institut für Experimentelle Kernphysik, Karlsruher Institut für Technologie, 76131 Karlsruhe, Germany \\ ${ }_{9}^{9}$ Physikalisch-Technische Bundesanstalt, Bundesallee 100, 38116 Braunschweig, Germany \\ ${ }^{10}$ Physics Department, Lancaster University, Lancaster LA1 4YB, UK \\ ${ }_{11}$ Department of Physics, Center for Experimental Nuclear Physics and Astrophysics, University of Washington, Seattle, WA 98195, USA \\ 12 Department of Radiation Oncology, Mayo Clinic, Rochester, MN 55905, USA \\ ${ }^{13}$ School of Physics and Astronomy, Queen Mary, University of London, London E1 4NS, UK
}

Received: 4 October 2015 / Accepted: 19 February 2016 / Published online: 29 February 2016

(C) The Author(s) 2016. This article is published with open access at Springerlink.com

\begin{abstract}
The $\alpha$-particle light response of liquid scintillators based on linear alkylbenzene (LAB) has been measured with three different experimental approaches. In the first approach, $\alpha$-particles were produced in the scintillator via ${ }^{12} \mathrm{C}(n, \alpha){ }^{9} \mathrm{Be}$ reactions. In the second approach, the scintillator was loaded with $2 \%$ of ${ }^{\text {nat }} \mathrm{Sm}$ providing an $\alpha$ emitter, ${ }^{147} \mathrm{Sm}$, as an internal source. In the third approach, a scintillator flask was deployed into the water-filled SNO+ detector and the radioactive contaminants ${ }^{222} \mathrm{Rn},{ }^{218} \mathrm{Po}$ and ${ }^{214} \mathrm{Po}$ provided the $\alpha$-particle signal. The behavior of the observed $\alpha$-particle light outputs are in agreement with each case successfully described by Birks' law. The resulting Birks parameter $k B$ ranges from $(0.0066 \pm 0.0016)$ to $(0.0076 \pm 0.0003) \mathrm{cm} / \mathrm{MeV}$. In the first approach, the $\alpha$ particle light response was measured simultaneously with the light response of recoil protons produced via neutronproton elastic scattering. This enabled a first time a direct comparison of $k B$ describing the proton and the $\alpha$-particle response of $\mathrm{LAB}$ based scintillator. The observed $k B$ values describing the two light response functions deviate by more than $5 \sigma$. The presented results are valuable for all current and future detectors, using LAB based scintillator as target, since they depend on an accurate knowledge of the scintillator response to different particles.
\end{abstract}

\footnotetext{
a e-mail: bkrosigk@physics.ubc.ca
}

\section{Introduction}

Over the last decades, liquid scintillation detectors gained great importance in neutrino and astroparticle physics. This is due to their capability to detect the charged secondary particles of neutrino interactions down to energies of a few $\mathrm{keV}$ in realtime and due to the easy scaling to large target masses. The scintillation light output $L$ scales with the energy of the charged particle, providing valuable kinematic information. In addition, the observed light output of the scintillator at a certain particle energy decreases with increasing ionization density. This effect, known as ionization quenching, allows to discriminate heavily ionizing particles, like protons and $\alpha$-particles, from electrons. The energy dependent, quenched scintillation light output can be described analytically by Birks law [1,2],

$L(E)=S \cdot \int_{0}^{E} \frac{\mathrm{d} E}{1+k B\left(\frac{\mathrm{d} E}{\mathrm{~d} x}\right)}$,

where $\mathrm{d} E / \mathrm{d} x$ is the specific energy loss. $k B$ denotes Birks' parameter and $S$ is a scaling parameter, which is associated with the scintillation efficiency. For fast electrons, which have a small $\mathrm{d} E / \mathrm{d} x$, Eq. 1 approximates the proportionality

$L_{e}(E)=S \cdot E$,

where the index $e$ refers to electrons [2]. For many of the standard organic liquid and plastic scintillators, a linear elec- 
tron scintillation light response has been observed down to about $100 \mathrm{keV}$ [3-7]. This includes also LAB based scintillators [6,7]. At lower energies, $\mathrm{d} E / \mathrm{d} x$ is increased with respect to fast electrons and $L$ rises non-linearly with $E$. The small non-linearity results in an energy offset, if Eq. 2 is used to describe the electron light output function. This offset was experimentally determined to be $\lesssim 5 \mathrm{keV}[4,6,8]$. This quasilinear behavior of scintillation light induced by electrons is typically taken advantage of in order to calibrate the light output scale in ionization quenching measurements. Often a scale in units of electron-equivalent energy is chosen and thus $S=1$. This scale is used throughout this article for $L$.

With the evolution of both, detector technology and understanding of the scintillator properties, experiments using scintillation detectors grew in the second half of the last century from small-scale to multi-tonne detectors. Several large-scale liquid scintillator detectors with up to $1 \mathrm{kt}$ scintillator mass are currently operational worldwide and multikilotonne devices are being designed. However, despite the great progress in understanding the mechanisms of liquid scintillation, some fundamental questions still remain unanswered. One of these questions is whether the same value of $k B$ can describe the light response of a certain organic scintillator to different ions [9], a question that was raised already in the original works by Birks [2] upon the observation of $k B$ (proton) $\neq k B(\alpha)$. However, since no consistent behavior was observed, no conclusive answer was found at that time. And also to date, this question still remains unresolved. As already discussed in the seminal work of Birks [1,2], the increased complexity of the reaction kinetics in multicomponent liquid scintillators compared with organic plastic scintillators or inorganic scintillators makes a unique answer to this question impossible. Therefore, individual investigations are crucial for the wide range of operational and future large-scale liquid scintillator detectors like Daya Bay [10], RENO [11], SNO+ [12], JUNO [13], RENO-50 [14], LENS [15] and HANOHANO [16]. All of these experiments use, or consider to use, LAB based scintillator.

Section 2 of this article describes the measurement and analysis of $\alpha$-particle quenching in LAB based scintillators using fast neutrons. This experiment is referred to as the "neutron beam experiment". The measurements were carried out at the PTB ${ }^{1}$ Ion Accelerator Facility (PIAF) $[17,18]$, which provides a neutron beam with a continuous energy distribution. The individual neutron energies are derived from time-of-flight (TOF) measurements. $\alpha$-particles are produced inside the scintillator through $(n, \alpha)$ reactions and outgoing $\alpha$-particles with an energy known from kinematic calculations are identified within the data. The obtained quenching results are compared to the results of the proton quenching measurements published in [19], which make use of $n-p$

\footnotetext{
${ }^{1}$ Physikalisch-Technische Bundesanstalt.
}

elastic scattering in the scintillator. Both measurements were taken simultaneously and with the same scintillation detector.

Section 3 presents the second $\alpha$-quenching experiment, which uses samarium-loaded LAB based scintillator, referred to as the "samarium experiment". The scintillator was loaded for this experiment with $2 \%{ }^{\text {nat }} \mathrm{Sm}$ at $\mathrm{BNL}^{2}$ and the measurement was carried out at HZDR. ${ }^{3}$ The isotope ${ }^{147} \mathrm{Sm}$ is an $\alpha$-emitter with a $Q$-value of 2.3105(11) MeV [20], providing an internal $\alpha$-source.

Section 4 describes the third measurement of $\alpha$-particle quenching in LAB based scintillator, referred to as the "bucket source experiment". In this case, the results were obtained from a 11 sample deployed within an acrylic container, the bucket, into the water-filled SNO+ detector. Contaminations of the scintillator by the $\alpha$-emitters ${ }^{222} \mathrm{Rn},{ }^{218} \mathrm{Po}$ and ${ }^{214} \mathrm{Po}$ are used as internal $\alpha$-sources with particle energies of 5.49, 6.00 and $7.69 \mathrm{MeV}$, respectively.

All three independent experiments were conducted with small liquid scintillator volumes, using charged particles produced in the scintillator itself. Furthermore all three experiments had a comparable sensitivity to UV light. This is important because a small fraction of Cherenkov light is always emitted in addition to the scintillation light, when an electron with an energy above the Cherenkov threshold of about $166 \mathrm{keV}$ traverses the LAB based scintillator. In references [6,7] it is shown that the additional Cherenkov light slightly changes the gradient of the linear relation between electron energy and light output and thus the calibration to electron-equivalent energy. Throughout this article, the observed pulse-height $P H$ is calibrated in units of electron-equivalent energies, including the scintillation and Cherenkov photons.

The scintillator consists of the solvent $\mathrm{LAB}$, the primary fluor 2,5-diphenyloxazole (PPO) and the secondary fluor, if any, p-bis-(o-methylstyryl)-benzene (bis-MSB). Bis-MSB acts as a wavelength shifter to achieve a better match of the spectral distribution of the scintillation light and the sensitivity of the photocathode. The LAB solvent was obtained from Petresa Canada Inc. [21] with an average stoichiometric composition of $\mathrm{C}_{17.1} \mathrm{H}_{28.3}$.

A direct comparison of the $\alpha$-particle quenching measurement results from these three experiments is presented in Sect. 5. Section 6 provides a direct comparison of the quenching parameters $k B$ for protons and $\alpha$-particles, determined in a simultaneous measurement using neutron-induced reactions in the scintillator. The proton quenching data were already published earlier [19]. A summary of the article and an outlook is given in Sect. 7.

\footnotetext{
${ }^{2}$ Brookhaven National Laboratory.

${ }^{3}$ Helmholtz-Zentrum Dresden-Rossendorf.
} 


\section{Measurements of $\alpha$-particle quenching using fast neutrons}

In the neutron beam experiment, the $\alpha$-particle quenching in two deoxygenated, ternary LAB based scintillators is determined from the same data sets used for the proton quenching analysis [19]. The respective scintillator samples are $\mathrm{LAB}+2 \mathrm{~g} / \mathrm{l} \mathrm{PPO}+15 \mathrm{mg} / \mathrm{l}$ bis-MSB and LAB $+3 \mathrm{~g} / \mathrm{l}$ $\mathrm{PPO}+15 \mathrm{mg} / \mathrm{l}$ bis-MSB. The only difference in these two samples is the PPO concentration. The analysis of the $\alpha$ particle quenching has higher demands on the $\mathrm{PH}$ resolution than the one of the proton quenching because structures caused by $\alpha$-particles from $(n, \alpha)$ reactions have to be discriminated from those resulting from other reactions. Thus the additional two binary LAB based scintillators without bis-MSB, used in [19], cannot be used for the analysis of the $\alpha$-particle quenching because they show a lower resolution than the ternary scintillators. The experimental setup, data acquisition and calibration is described in detail in [19]. Therefore only details specifically relevant for the analysis of the $\alpha$-particle quenching are discussed here.

\subsection{Experimental setup and data extraction}

The beam of fast neutrons was produced by bombarding a $3 \mathrm{~mm}$ Beryllium target with $19 \mathrm{MeV}$ protons from the CV28 cyclotron at PIAF. The neutrons resulting from ${ }^{9} \mathrm{Be}(p, n x)$ reactions have a continuous kinetic energy distribution from about 1 to $17 \mathrm{MeV}$ and are observed together with prompt $\gamma$-rays produced in inelastic interactions. The individual neutron energy $E_{n}$ is deduced from a measurement of the neutron TOF relative to the centroid TOF value of the prompt $\gamma$-peak together with the flight distance from the target to the scintillator volume.

The scintillation detector had an active volume of about $100 \mathrm{ml}$. It was observed by an XP2020Q PMT, which has an increased UV light sensitivity. All materials between scintillator and photocathode were UV transparent. An integrated and amplified charge signal was derived from the 9th dynode out of 12, avoiding a non-linear PMT gain and its pulse height $P H$ was measured using a peak sensing analog-todigital converter (ADC).

The high-voltage applied to the PMT, and thus the PMT gain, was increased for the $\alpha$-particle quenching and low energy proton quenching measurements, compared to the proton quenching measurements at energies above about $5 \mathrm{MeV}$ [19]. This achieves a better resolution of low $P H$ signals, which is especially important for the analysis of the highly quenched $\alpha$-particle light pulses. This mode is referred to as high gain $(\mathrm{HG})$ mode.

The data are stored in a $P H$ versus TOF matrix. From this matrix, $P H$ spectra are extracted by selecting a small TOF window around the TOF of interest and by projection of the selected events on the $P H$ axis. The $P H$ scale is calibrated with $\gamma$-ray sources yielding $L$ in units of electronequivalent energy. The TOF, and thus $E_{n}$, window is always made smaller than the $E_{n}$ window corresponding to the light output resolution $\Delta L$ at the respective particle energy.

\subsection{Calibration}

Three $\gamma$-ray sources were used for calibration: ${ }^{137} \mathrm{Cs},{ }^{22} \mathrm{Na}$ and ${ }^{207} \mathrm{Bi}$. They provide in total six $\gamma$-rays with different energies. Figure 1 shows the observed $P H$ distribution of ${ }^{207} \mathrm{Bi}$ as an example. The three ${ }^{207} \mathrm{Bi} \gamma$-rays lead to three sharp edges at which the Compton electrons have the maximum energy $E$. Since this energy is precisely known, the relation between $P H$ and electron energy is determined by fitting the simulated $P H$ distributions in the region around the Compton edges to the measured ones [19]. The simulation is performed with the code GRESP7 [22]. Despite small deviations at lower pulse-heights, the region around the Compton edges is very well described by the simulation. The deviations result mainly from a simplified description of surface effects within the GRESP7 simulation.

The described procedure yields six data points $P H_{i}=$ $P H\left(E_{i}\right)$, where $i=1-6$. A linear fit to these data points, with

$P H(E)=m \cdot E+a$,

results in the two calibration parameters $m=(343.4 \pm$ 1.1) channels/MeV and $a=(-9.0 \pm 2.0)$ channels for $\mathrm{LAB}+2 \mathrm{~g} / \mathrm{l} \mathrm{PPO}+15 \mathrm{mg} / \mathrm{l}$ bis-MSB . Using instead $\mathrm{LAB}+3 \mathrm{~g} / \mathrm{lPPO}+15 \mathrm{mg} / \mathrm{l}$ bis-MSB results in the param-

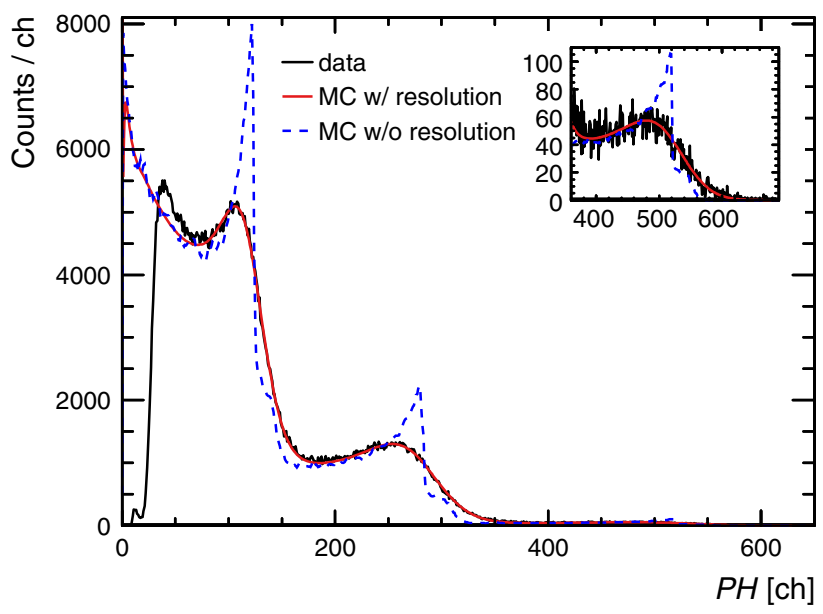

Fig. 1 Distribution of the pulse-height $P H$ measured in ADC channels with a ${ }^{207} \mathrm{Bi}$ calibration source. The data were taken with LAB + $2 \mathrm{~g} / \mathrm{l} \mathrm{PPO}+15 \mathrm{mg} / \mathrm{l}$ bis-MSB. Also shown is the Monte Carlo (MC) distribution before and after folding it with the detector resolution function. The third Compton edge at high $P H$ is shown enlarged in the inset 
eter values $m=(350.5 \pm 0.7)$ channels/MeV and $a=$ $(-11.1 \pm 2.1)$ channels.

The light output in units of electron-equivalent energy is finally obtained from Eqs. 2 and 3 and thus

$$
L(E)=\frac{P H-a}{m} .
$$

\subsection{Determination of the $\alpha$-particle response}

\subsubsection{Reactions producing $\alpha$-particles}

The beam neutrons reaching the sensitive volume mostly elastically scatter off protons in the scintillator producing a recoil proton energy distribution [19]. Neutrons that exceed a threshold energy of $6.19 \mathrm{MeV}$ can furthermore produce $\alpha$-particles via the reaction

$$
{ }^{12} \mathrm{C}(n, \alpha){ }^{9} \mathrm{Be}(\text { g.s. })
$$

with scintillator intrinsic ${ }^{12} \mathrm{C}$ nuclei. The maximum possible energy of the single $\alpha$-particle in the final state is unambiguously related to the energy $E_{n}$ of the incoming neutron. Above $E_{n}=8.81$ and $8.29 \mathrm{MeV}$, respectively, two further reactions contribute to the production of $\alpha$-particles:

$$
\begin{array}{r}
{ }^{12} \mathrm{C}\left(n, \alpha^{\prime}\right){ }^{9} \mathrm{Be}^{*} \rightarrow \quad n+{ }^{8} \mathrm{Be} \\
\downarrow \\
\\
{ }^{12} \mathrm{C}\left(n, n^{\prime}\right){ }^{12} \mathrm{C}^{*} \rightarrow \quad \alpha+{ }^{8} \mathrm{Be} \\
\downarrow \\
\\
\\
2 \alpha .
\end{array}
$$

These reactions have more than one $\alpha$-particle in the final state and it is not possible to identify $\alpha$-particles of known energy. They thus contribute to the background spectrum.

Natural background, for instance from ambient $\gamma$-radiation and scintillator internal impurities, was measured in the absence of calibration sources and beam neutrons and subtracted from data.

\subsubsection{Monte Carlo simulations}

The simulation of light yield distributions resulting from incoming neutrons is performed with the Monte Carlo (MC) code NRESP7 [23]. The non-linearly rising light output from different charged particles is simulated using a set of predefined light output functions, which are stored in an external file and iteratively adapted to the data. NRESP 7 describes the $\alpha$-particle light output function by two analytic expressions:

$$
\begin{aligned}
& L_{\alpha}(E)=c_{0} E^{c_{1}} \quad E<6.76 \mathrm{MeV} \\
& L_{\alpha}(E)=c_{2}+c_{3} E \quad E \geq 6.76 \mathrm{MeV}
\end{aligned}
$$

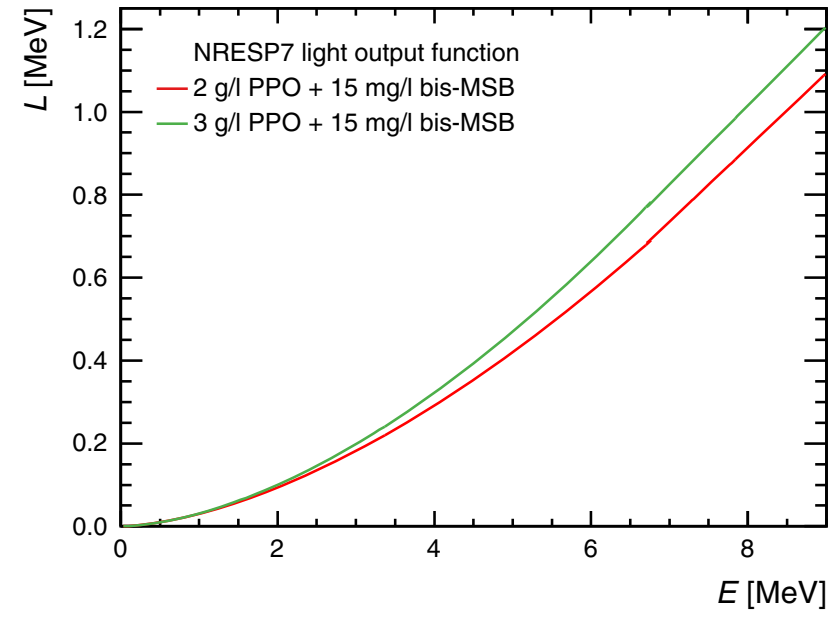

Fig. 2 Light output $L$ as function of kinetic energy $E$ used in NRESP7 to simulate $\alpha$-particle events in LAB $+2 \mathrm{~g} / \mathrm{lPPO}+15 \mathrm{mg} / \mathrm{l}$ bis-MSB and $\mathrm{LAB}+3 \mathrm{~g} / \mathrm{l} \mathrm{PPO}+15 \mathrm{mg} / \mathrm{l}$ bis-MSB . The two functions shown result from iterative adaptations of the simulated to the measured light yield distributions

The parameter values resulting from the last iteration are $c_{0}=0.030, c_{1}=1.640, c_{2}=-0.518, c_{3}=0.179$ for $\mathrm{LAB}+2 \mathrm{~g} / \mathrm{l} \mathrm{PPO}+15 \mathrm{mg} / \mathrm{l}$ bis-MSB and $c_{0}=$ $0.031, c_{1}=1.689, c_{2}=-0.505, c_{3}=0.190$ for $\mathrm{LAB}+3 \mathrm{~g} / \mathrm{PPO}+15 \mathrm{mg} / \mathrm{l}$ bis-MSB . The respective functions are shown in Fig. 2.

The determination of the proton light output function is possible without the knowledge of the specific $\alpha$-particle light output function, which is not the case vice versa. The reason is that the individual regions of interest used for the proton analysis nearly exclusively contain proton events [19]. Instead, the regions of interest for the $\alpha$-particle analysis are not dominated by $\alpha$-particle, but also by proton events. Hence, the proton analysis was conducted first.

Figure 3 shows the simulated light yield distributions resulting from the reactions (5)-(7) as well as their sum. The calibration parameters for $\mathrm{LAB}+2 \mathrm{~g} / \mathrm{l} \mathrm{PPO}+15 \mathrm{mg} / \mathrm{l} \mathrm{bis}-$ MSB (see Sect. 2.2) are considered and an incoming neutron energy of $E_{n}=(11.0 \pm 0.4) \mathrm{MeV}$ is assumed. The width of the rectangular neutron energy distribution corresponds to the TOF window width used for the extraction of the respective data spectrum. The edge visible around $430 \mathrm{keV}$ results from $\alpha$-particles which are produced in the reaction (5) and emitted at forward angles. The corresponding $\alpha$ particle energy can be calculated using simple two-particle kinematics, assuming ${ }^{12} \mathrm{C}$ being at rest. For the given example with $E_{n}=11.0 \mathrm{MeV}$, the $\alpha$-particle energy is $5.14 \mathrm{MeV}$. In Fig. 3 the high energy edge lies outside the region covered by events from the break-up reactions (6) and (7). This enables a precise assignment of a known $\alpha$-particle energy to a particular value of $L$. 


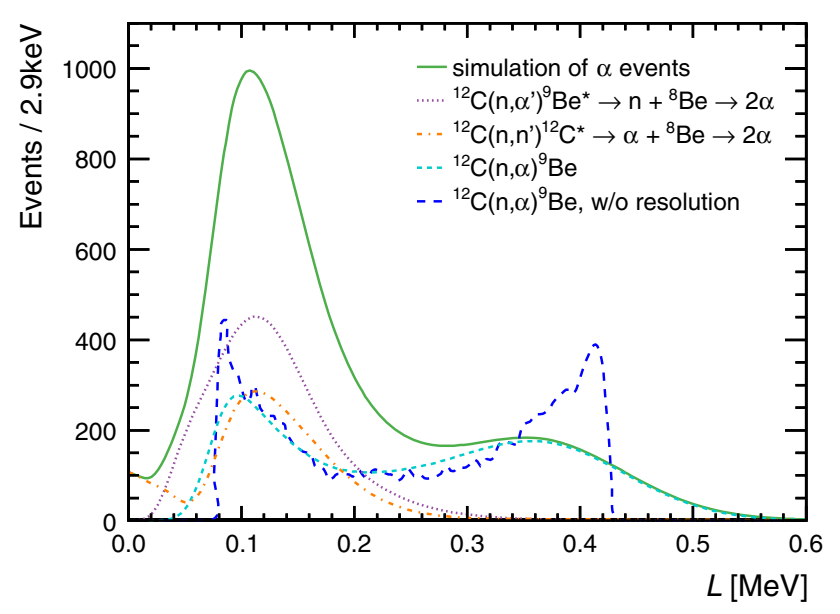

Fig. 3 Simulated distributions of the total light yield $L$ in units of electron-equivalent energy in LAB $+2 \mathrm{~g} / \mathrm{l} \mathrm{PPO}+15 \mathrm{mg} / \mathrm{l}$ bis-MSB due to $\alpha$-particles from reactions (5)-(7). The energy of the incoming neutrons ranges from 10.6 to $11.4 \mathrm{MeV}$ with a uniform energy distribution. Additionally shown is the $L$ distribution due to reaction (5), induced by mono-energetic $11 \mathrm{MeV}$ neutrons and without considering the detector resolution. The sharp high energy edge at about $430 \mathrm{keV}$ is produced by $\alpha$-particles with $E_{\alpha}=5.14 \mathrm{MeV}$

The advantage of NRESP7 is the relatively detailed description of the $(n, \alpha x)$ reactions on carbon nuclei, compared to e.g. standard Geant 4 . There is no Geant 4 version available, or dedicated class for the use with Geant 4 , which contains for instance a model for the various $3 \alpha$-break-up channels. ${ }^{4}$ The $(n, \alpha x)$ reactions are of particular relevance for the determination of the light output function for $\alpha$-particles. It should be noted, however, that independent of the code, the individual differential neutron cross sections on carbon are still not well-known [23]. The available evaluated data files, such as ENDF, JENDL or JEFF, only contain differential level cross sections for ${ }^{12} \mathrm{C}(n, \alpha){ }^{9} \mathrm{Be}^{*}$ or double-differential $\alpha$-emission cross sections $\mathrm{d} \sigma /(\mathrm{d} \Omega \mathrm{d} E)$, i.e. the statistical average of the $\alpha$-particle emission over the individual $3 \alpha$-break-up reactions. For modeling the scintillation detector, however, the correlations of the $\alpha$-particles in an individual reaction are required, i.e. $\mathrm{d} \sigma /\left(\mathrm{d} E_{\alpha 1} \mathrm{~d} E_{\alpha 2} \mathrm{~d} E_{\alpha 3}\right)$. Although the ENDF format basically has the capability to represent these data, no information on these channels is available for ${ }^{12} \mathrm{C}$. The shortcoming regarding the individual differential neutron cross sections on carbon results in an uncertainty on the shape of the $\alpha$-particle energy distributions. This uncertainty has to be considered for the determination of the position of the $\alpha$-particle edge from reaction (5) within the total light yield distribution.

\footnotetext{
$\overline{4}$ Work is underway at CIEMAT, Madrid, to migrate the NRESP7 model to Geant4.
}

\subsubsection{Comparison with data}

The simulated total light yield distribution, resulting from $\alpha$ particle events and non- $\alpha$-particle events, is shown in Fig. 4 together with the data spectrum. The $\alpha$-particle edge in the given example is well reproduced by the simulation. The small mismatch between simulation and data, observed in Fig. 4, is due to the fact that NRESP7 does not model interactions of de-excitation $\gamma$-rays, which result mostly from the first excited state in ${ }^{12} \mathrm{C}$ at $4.439 \mathrm{MeV}$, populated by inelastic neutron scattering. Instead, it is assumed that these events are suppressed by pulse-shape discrimination (PSD). Because of the poor $n / \gamma$-PSD properties of LAB based scintillators, however, $\gamma$-induced events could not be separated from neutron-induced events in the data [19]. Hence, a direct comparison of experimental and simulated light yield distributions is difficult in regions where $\gamma$-induced events contribute significantly. Fortunately, the Compton edge from this $\gamma$-radiation lies far outside the region of the observed edge attributed to $\alpha$-particles. This is obvious from a comparison with the inset in Fig. 1, which shows the edge position from $\gamma$-rays with an energy of $1.770 \mathrm{MeV}$. Within the $\alpha$ particle edge region itself, no data excess is visible since the $\alpha$-particle event simulation is normalized individually to data in this region to reduce the sensitivity to the insufficient knowledge of the neutron cross sections on carbon. As a consequence, the $\alpha$-particle event yield is slightly overestimated in the presence of background events. The location of the edge, though, is not affected, as long as the background is smooth as is the case discussed here.

The analysis of multiple, measured light yield distributions induced by neutrons with different $E_{n}$ provides a set

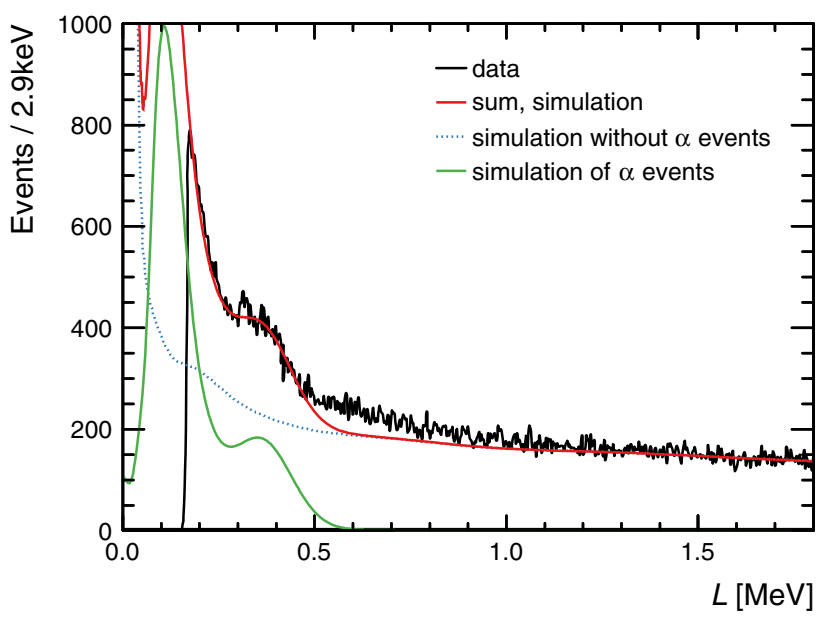

Fig. 4 Measured and simulated distributions of the total light yield $L$ in units of electron-equivalent energy in LAB $+2 \mathrm{~g} / \mathrm{l} \mathrm{PPO}+15 \mathrm{mg} / \mathrm{l}$ bis-MSB induced by neutrons with $E_{n}=(11.0 \pm 0.4) \mathrm{MeV}$. The contributions of $\alpha$-particle and non- $\alpha$-particle (mainly recoil proton) events to the total simulated $L$ spectrum are shown individually 
of data points $L_{i}$. This is, of course, only possible if the $\alpha$-particle edge is identified within the total light yield distribution, which is dominated by recoil proton events. The ${ }^{12} \mathrm{C}(n, \alpha){ }^{9} \mathrm{Be}$ reaction leads to an observable structure in the $L$ spectra (see Fig. 4), as soon as the $\alpha$-particle light output is high enough to overcome the detector threshold. This is the case for $E_{n} \gtrsim 9.5 \mathrm{MeV}$ in the investigated data sets. At $E_{n} \gtrsim 11.5 \mathrm{MeV}$ the two additional reactions, (6) and (7), dominate the $P H$ spectrum resulting from all $\alpha$-particle events. The $\alpha$-particle edge with known energy can not be located anymore until $E_{n} \approx 14 \mathrm{MeV}$. At these high neutron energies, the maximum $\alpha$-particle energy resulting from reaction (5) is high enough to induce a light output well outside the light yield distributions from the other two reactions. Finally, the number of light yield distributions with an observable $\alpha$-particle edge also depends on the $P H$ resolution.

The sources of systematic uncertainties are the $P H$ calibration, the TOF measurement, the PMT gain and the MC simulations. The uncertainties on the calibration parameters $m$ and $a$ are given in Sect. 2.2. These uncertainties, as well as all uncertainties on the TOF measurement and the PMT gain, are the same as for the measurement of the light production by protons and are discussed in [19]. The MC uncertainty, however, differs and is significantly larger for $\alpha$-particles. It results mainly from the uncertainty on the differential $(n, \alpha x)$ cross sections for carbon, the effect of photon induced events not covered by the simulation and the residual uncertainty on the determined proton light output function. These uncertainties within the MC manifest in the dependence of the $\alpha$-particle edge position on the $L$ interval chosen for the fit of the MC to data. For this reason, the $L$ interval around the edge position is systematically varied and the average edge position is determined. The maximum deviation from the average position observed in all analyzed light yield distributions is $\pm 26 \mathrm{keV}$.

\subsubsection{Results}

Figure 5a, b show the $\alpha$-particle data points $L_{i}$, determined as described above for LAB $+2 \mathrm{~g} / \mathrm{l} \mathrm{PPO}+15 \mathrm{mg} / \mathrm{l}$ bis-MSB and $\mathrm{LAB}+3 \mathrm{~g} / \mathrm{l} \mathrm{PPO}+15 \mathrm{mg} / \mathrm{l}$ bis-MSB, respectively. The evaluated $\alpha$-particle energies partly differ for the two scintillator samples, since besides the fluor concentration, the experimental settings like the amplification were slightly different in the two measurements. Both aspects affect the light output resolution.

The $\alpha$-particle light output function $L(E)$ is determined by a $\chi^{2}$ fit of Eq. 1 to the data points. In this fit, all systematic uncertainties mentioned in Sect. 2.3.3 are included as nuisance parameters (see [19] for details). The specific energy loss in the respective scintillator is calculated using SRIM [24]. An uncertainty on the $(\mathrm{d} E / \mathrm{d} x)(E)$ calculation of

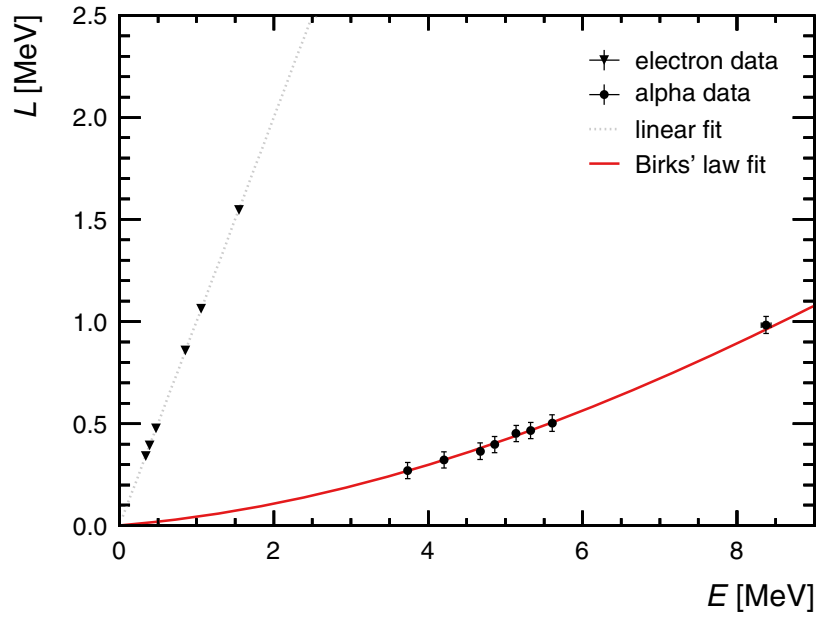

(a) $\mathrm{LAB}+2 \mathrm{~g} / \mathrm{lPPO}+15 \mathrm{mg} / \mathrm{l}$ bis-MSB

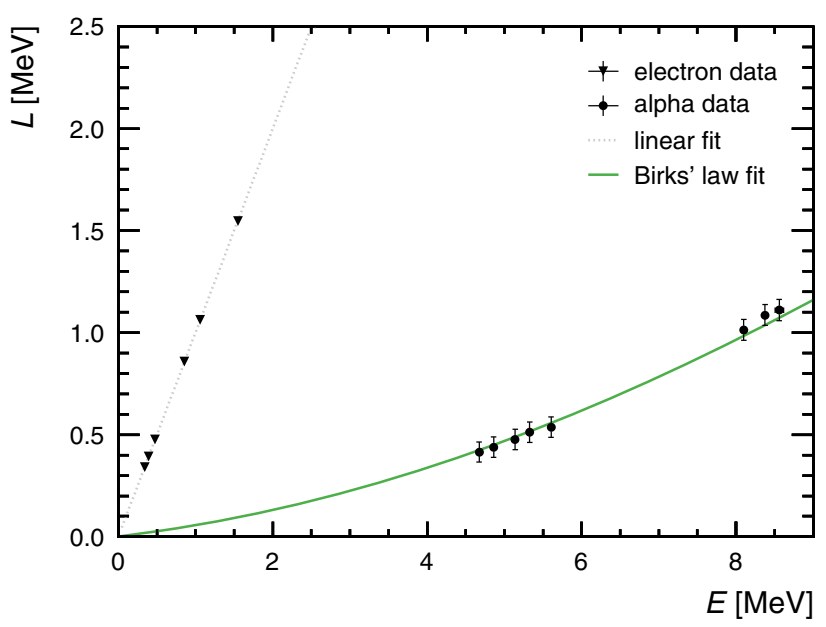

(b) $\mathrm{LAB}+3 \mathrm{~g} / \mathrm{lPPO}+15 \mathrm{mg} / \mathrm{lbis}-\mathrm{MSB}$

Fig. 5 Light output $L$ in units of electron-equivalent energy as function of kinetic energy $E$. $L$ is shown for electrons and $\alpha$-particles in LAB scintillator with $15 \mathrm{mg} / \mathrm{l}$ bis-MSB and $2 \mathrm{~g} / \mathrm{l}$ (a) or $3 \mathrm{~g} / \mathrm{l}$ PPO (b). The $\alpha$ particle data is fitted with Eq. 1 including systematic effects as nuisance parameters. In the shown total uncertainties, the single contributions are added quadratically

$2 \%$ is considered $[19,25]$. The resulting best fit values for the Birks parameter are $k B=(0.0076 \pm 0.0003) \mathrm{cm} / \mathrm{MeV}$ (see Fig. 5a) and $k B=(0.0071 \pm 0.0003) \mathrm{cm} / \mathrm{MeV}$ (see Fig. 5b). The corresponding $\chi^{2}$ values per degree of freedom are 0.73 and 1.36 , respectively.

These results, obtained using the two LAB samples which only differ in the concentration of the primary fluor, agree within $1.2 \sigma$. This is in line with the theoretical expectation [2] as well as the observation in [19] that the fluors do not significantly affect the ionization quenching parameters at the given concentrations. Ionization quenching is, according to [2], a primary process and thus competing with the excitation of the solvent and not with the energy transfers from 
the solvent to the solute, which are referred to as secondary processes.

\section{Measurement of $\alpha$-particle quenching using samarium-loaded scintillator}

While the $\alpha$-particle kinetic energy corresponding to an observed light output is laborious to access in the neutron beam experiment presented in Sect. 2, its determination is straight forward observing the peak from an $\alpha$-source with known $\alpha$-particle energy. This requires the use of an internal $\alpha$-source to avoid energy losses within the source carrier and the escape of scintillation light produced near the scintillator surface. For this reason, LAB with $2 \mathrm{~g} / \mathrm{l} \mathrm{PPO}$ was loaded at BNL with $2 \%$ mass fraction of ${ }^{\text {nat }} \mathrm{Sm}$ which contains the $\alpha$ emitter ${ }^{147} \mathrm{Sm}$ with a natural abundance of $14.99(18) \%$. This isotope decays into ${ }^{143} \mathrm{Nd}$ with a half-life of $1.06 \times 10^{11} \mathrm{y}$ [20]. The kinetic energy of the $\alpha$-particles is $2.248 \mathrm{MeV}$, which is lower than the energies accessible by ${ }^{12} \mathrm{C}(n, \alpha){ }^{9} \mathrm{Be}$ reactions. The samarium experiment is thus complementary to the previously described measurement. The fact that only one $\alpha$-particle energy is observable enhances the sensitivity to the respective energy compared to the situation with multiple energy peaks overlapping in the visible spectrum. To ensure that no background resulting from the radioactivity of the detector and environment fakes an $\alpha$-peak, an independent measurement with unloaded LAB $+2 \mathrm{~g} / \mathrm{l} \mathrm{PPO}$ was conducted.

The high concentration of Sm increases the density of the full liquid scintillator cocktail to about $0.99 \mathrm{~g} / \mathrm{cm}^{3}$. As no direct measurement of the density was done at the time, it was calculated assuming a LAB density of $0.86 \mathrm{~g} / \mathrm{cm}^{3}$ for the LAB cocktail [21] and a Sm density of $7.54 \mathrm{~g} / \mathrm{cm}^{3}$ [26].

\subsection{Experimental setup}

To measure the $\alpha$-particle light output, the liquid scintillator was filled into a UV transparent cylindrical fused silica cuvette $^{5}$ with a length of $100 \mathrm{~mm}$ and a diameter of $19 \mathrm{~mm}$. The cuvette can thus hold about $28 \mathrm{ml} \mathrm{Sm-loaded} \mathrm{LAB.}$ To avoid oxygen in the cuvette, the filling was performed in a nitrogen atmosphere. The LAB solution itself was deoxygenated by bubbling it for $30 \mathrm{~min}$ with gaseous nitrogen. The filled cuvette was covered with Teflon (PTFE) tape to improve the reflectivity at the cuvette walls. Each front of the cuvette was coupled to an R2059-01 PMT using UV transparent Baysilone M 200.000 silicon grease. This type of phototube is equipped with a fused silica window and provides an increased UV sensitivity. An active voltage divider guaranteeing long term stability in gain was used for the

${ }^{5}$ Hellma Analytics. high voltage supply of the PMTs [27]. The setup with two PMTs operating in coincidence mode enabled the suppression of thermal noise and improves the position dependent resolution. The cuvette was covered with aluminum foil and a heat-shrink tube, to screen it from light, and was tested to be light tight.

The signal of each PMT was split. One signal was directed to a fast Acqiris DC-282 digitizer, the other one was fed into an Ortec 584 constant fraction discriminator (CFD). The CFD threshold was set close to the electronic noise level, to allow the detection also of small pulses. The signals from the CFD were fed into a fast coincidence unit with a coincidence time window of $50 \mathrm{~ns}$. In case the coincidence condition was fulfilled, data taking was triggered and the time dependent, digitalized pulse was stored. The coincidence events were stored event-by-event. Each stored pulse was subsequently integrated, providing the $P H$ of the event in arbitrary units (arb.u.). The $P H$ scale is calibrated with standard $\gamma$-ray sources.

\subsection{Calibration}

Three different sources were used for calibration: ${ }^{137} \mathrm{Cs},{ }^{60} \mathrm{Co}$ and ${ }^{166 \mathrm{~m}} \mathrm{Ho}$. The latter two sources provide several $\gamma$-rays, which are too close in energy to be resolved with the given setup and scintillator. The simulation cannot be fitted to the data spectrum around each Compton edge independently. This instance is demonstrated in Fig. 6 for the two dominant $\gamma$-rays of ${ }^{60} \mathrm{Co}$ with an energy of 1.333 and $1.173 \mathrm{MeV}$. Note that a simplified MC simulation is used for this experiment which does not consider multiple scattering, any other

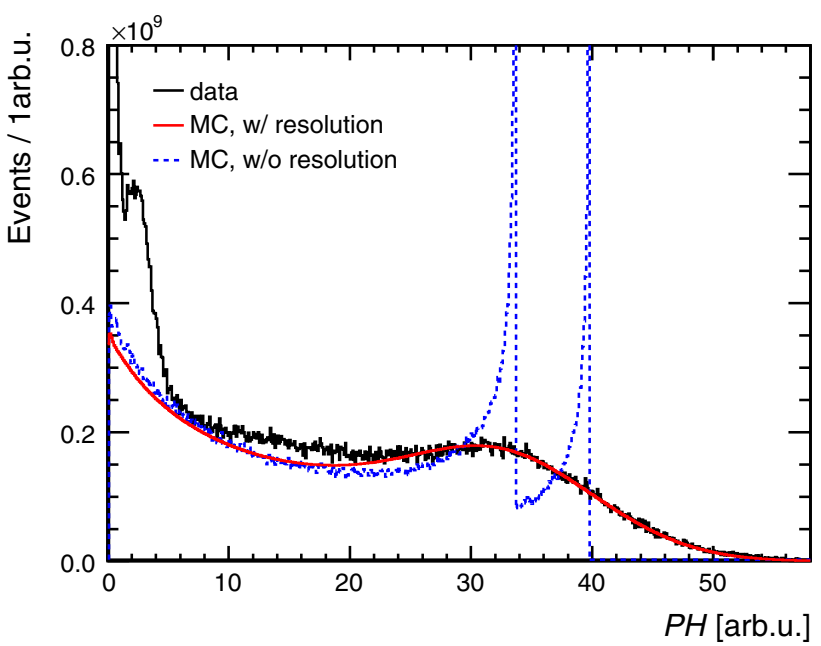

Fig. 6 Distribution of the pulse-height $P H$ measured with a ${ }^{60} \mathrm{Co}$ calibration source. The data were taken with $\mathrm{LAB}+2 \mathrm{~g} / \mathrm{l} \mathrm{PPO}+2 \%$ ${ }^{\text {nat }} \mathrm{Sm}$. The peak between (1-6) arb.u. is due to the $\alpha$-decay of ${ }^{147} \mathrm{Sm}$. Also shown is the Monte Carlo (MC) $\mathrm{PH}$ distribution before and after folding it with the detector resolution function 
interaction than Compton scattering, secondary photons or surface effects. The only detector effect considered is the $P H$ resolution. This treatment does not fully reproduce the data below the Compton edges including the distinct peak between (1-6) arb.u. from the $\alpha$-decay of ${ }^{147} \mathrm{Sm}$. However, the region of interest for the calibration is the region around the Compton edges, which is well-described by this calculation.

As a consequence of the unresolved single Compton edges, only the weighted mean value of the $\gamma$-energies is used per source, i.e. $E_{\gamma}=1.041 \mathrm{MeV}$ for ${ }^{60} \mathrm{Co}$ and $E_{\gamma}=0.571 \mathrm{MeV}$ for ${ }^{166 \mathrm{~m}} \mathrm{Ho}$. In total three calibration data points $P H_{i}$ are thus accessible. A fit of Eq. 3 to these data points results in the calibration parameters $m=$ (38.1 \pm 1.8$)$ arb.u./MeV and $a=(-2.8 \pm 1.2)$ arb.u. using Sm-loaded LAB and $m=(60.0 \pm 9.6)$ arb.u. $/ \mathrm{MeV}$ and $a=(-2.7 \pm 0.4)$ arb.u. using unloaded LAB.

\subsection{Determination of the ${ }^{147} \mathrm{Sm} \alpha$-peak position}

The calibrated background distribution measured with unloaded LAB is shown in Fig. 7. It is expected to mainly consist of events induced by $\beta$-particles and $\gamma$-rays of the natural ${ }^{238} \mathrm{U}$ and ${ }^{232} \mathrm{Th}$ decay chains. This natural background reveals no distinct peak structure and has a rate of $(13.76 \pm 0.02) 1 / \mathrm{s}$, observed over the full dynamic measurement range. The background rate above $75 \mathrm{keV}$, the detection threshold within the measurement with Sm-loaded LAB, amounts to $(8.91 \pm 0.01) 1 / \mathrm{s}$.

The light yield distribution measured with Sm-loaded LAB is shown in Fig. 8. The observed event rate is (75.80 \pm $0.04) 1 / \mathrm{s}$ before subtraction of the natural background and

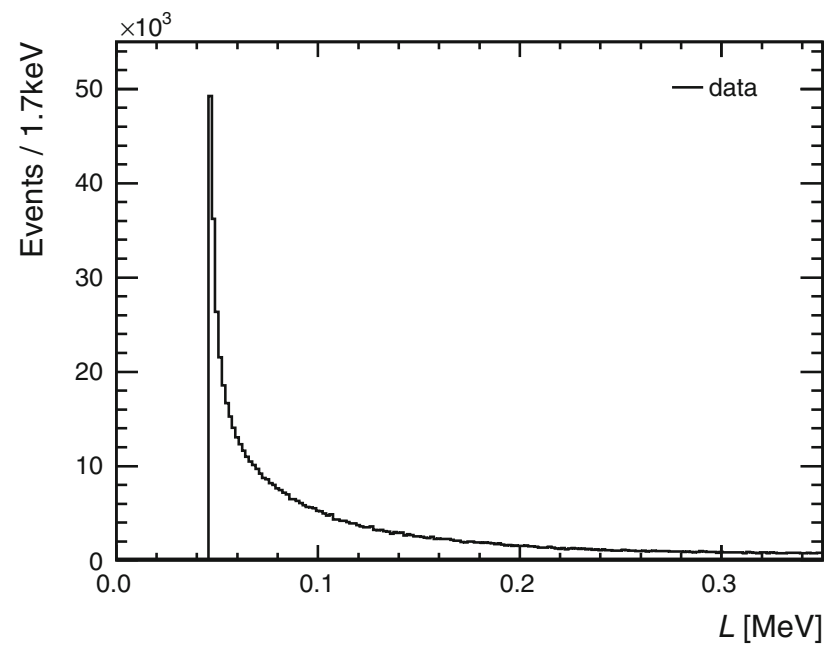

Fig. 7 Distribution of the total light yield $L$ in units of electronequivalent energy of background events measured with LAB $+2 \mathrm{~g} / \mathrm{l}$ PPO. The background is dominated by $\beta$ and $\gamma$ events from the natural radioactivity of the detector material and from ambient $\gamma$-radiation

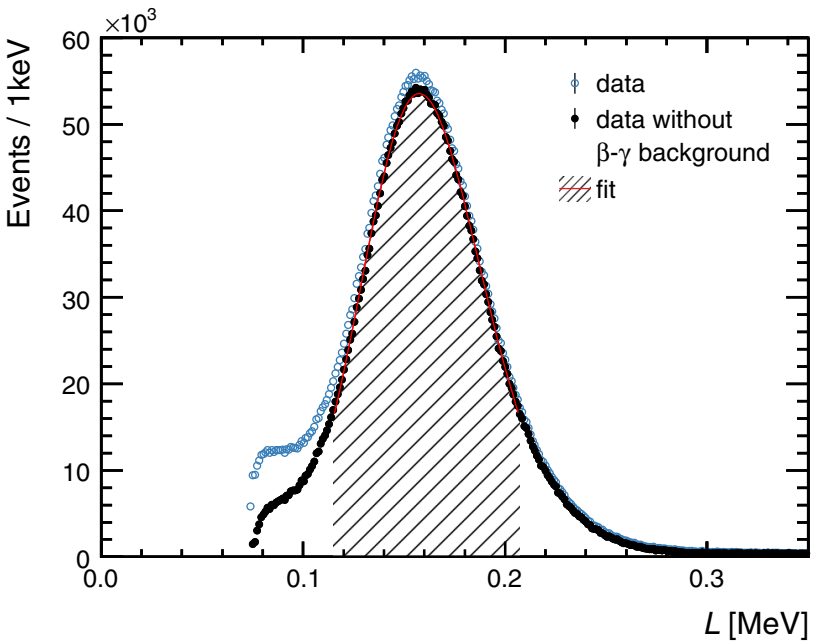

Fig. 8 Distribution of the total light yield $L$ in units of electronequivalent energy measured with $\mathrm{LAB}+2 \mathrm{~g} / \mathrm{lPO}+2 \%{ }^{\text {nat }} \mathrm{Sm}$ before and after background subtraction. This background was measured with unloaded LAB. The peak results from the $\alpha$-decay of ${ }^{147} \mathrm{Sm}$ and is fitted with an asymmetric Gaussian distribution

(66.89 \pm 0.04$) 1 / \mathrm{s}$ after subtraction of the natural background. The ${ }^{147} \mathrm{Sm}$ activity in $28 \mathrm{ml}$ of $2 \%{ }^{\text {nat }} \mathrm{Sm}$-loaded LAB is about 69.7 Bq. The observed ${ }^{147} \mathrm{Sm}$ decay rate is lower than the ${ }^{147} \mathrm{Sm}$ activity mainly due to a finite detection efficiency.

Note that the bin widths in Figs. 7 and 8 vary due to the different calibration parameters. The difference has been accounted for within the background subtraction. The incomplete background reduction in Fig. 8 is expected, since the loaded LAB cocktail is expected to have a higher level of impurities from the $\mathrm{U}$ and $\mathrm{Th}$ chain. Though ${ }^{\text {nat }} \mathrm{Sm}$ itself also contains another unstable isotope, ${ }^{148} \mathrm{Sm}$, with an abundance similar to the one of ${ }^{147} \mathrm{Sm}$, it does not significantly contribute to the measurement, since its half-life of $7 \times 10^{15} \mathrm{y}$ is nearly four orders of magnitude larger [20]. The chemical purity ${ }^{6}$ of the ${ }^{\text {nat }} \mathrm{Sm}$ used is $\geq 99 \%$ and no further rare Earth elements are expected to contribute to the background spectrum. Note that despite the residual natural background, a distinct $\alpha$-peak due to the decays of ${ }^{147} \mathrm{Sm}$ isotopes is clearly visible.

The value of $L$ corresponding to an $\alpha$-particle energy of $2.248 \mathrm{MeV}$ is obtained by locating the ${ }^{147} \mathrm{Sm} \alpha$-peak in Fig. 8 . Due to the residual background the peak is not fully Gaussian, but slightly asymmetric. Thus a generalization of the Gaussian distribution function,

$f(L)=\frac{e^{-\frac{(L-\xi)^{2}}{2 \omega^{2}}}}{\sqrt{2 \pi} \omega} \times \operatorname{erfc}\left[-\frac{s(L-\xi)}{\sqrt{2} \omega}\right]$,

is used, which allows for non-zero skewness $[28,29]$. The function " $\operatorname{erfc}(x)$ " is the complementary Gauss error function, which equals one if the distribution is symmetric. In

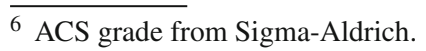


this case, the shape parameter $s$ is zero. The parameters $\xi$ and $\omega$ approximate the mean value and standard deviation of the distribution as it approximates a normal distribution. The best fit of Eq. 10 is used instead of a centroid calculation, since it revealed to be more robust and furthermore reproduces the peak region well, as shown in Fig. 8.

The $\chi^{2}$ value of the best fit distribution over the number of degrees of freedom amounts to 337/83. The position of the maximum of this distribution corresponds to the value of $L$ at $2.248 \mathrm{MeV}$ and is determined to be $L=(0.157 \pm 0.032) \mathrm{MeV}$. The given total uncertainty is obtained by error propagation, considering the uncertainty on the PMT gain, the uncertainty on the calibration parameters $m$ and $a$ (see Sect. 3.2) and the fit uncertainty. The PMT gain variations are measured to be less than $\pm 1 \%$. The fit uncertainty is $1 \times 10^{-4} \mathrm{MeV}$. According to the presented measurement, the $\alpha$-particle light output at $E=2.248 \mathrm{MeV}$ is quenched by a factor of $14.3 \pm 2.9$ compared to electrons. A $\chi^{2}$ fit of Birks' law Eq. 1 to the obtained data point yields $k B=(0.0066 \pm 0.0016) \mathrm{cm} / \mathrm{MeV}$. Also in this case, $(\mathrm{d} E / \mathrm{d} x)(E)$ is calculated using SRIM. This fit is only possible, since $L(0 \mathrm{MeV})=0 \mathrm{MeV}$, which fixes the fit at this point. The given total uncertainty on $k B$ is the quadratic sum of the individual contributions and includes a $10 \%$ uncertainty on the calculated Sm-LAB density. This uncertainty is chosen arbitrarily large and amounts to $\pm 0.0007 \mathrm{~cm} / \mathrm{MeV}$. Thus, an accurate measurement of the density can reduce the total uncertainty to $0.0014 \mathrm{~cm} / \mathrm{MeV}$. The data point and best fit function is presented and discussed in Sect. 5.

\section{Measurement of $\alpha$-particle quenching using scintillator internal radioactivity and the $\mathrm{SNO}+$ detector}

The third experiment discussed here is the bucket source experiment. It was conducted in 2008 during the transition phase from the SNO [30] to the SNO+ [12] experiment. In this phase, the entire $\mathrm{SNO} / \mathrm{SNO}+$ detector was filled with water. The results are presented here by courtesy of the $\mathrm{SNO}+$ collaboration.

\subsection{Experimental setup}

The SNO+ detector is installed in a barrel-shaped $34 \mathrm{~m}$ deep and $\leq 22 \mathrm{~m}$ wide cavity. The center of the detector is formed from a $12 \mathrm{~m}$ diameter acrylic vessel (AV) of $5 \mathrm{~cm}$ thickness, connected to the deck area via a cylindrical neck and supported by a hanging rope system. It is surrounded by a PMT support structure (PSUP), a $17.8 \mathrm{~m}$ diameter geodesic stainless steel frame holding more than 9400 Hamamatsu $8^{\prime \prime}$-R1408-PMTs. Each PMT is equipped with a reflective collar. This configuration yields a solid angle coverage of about $54 \%$ [30]. The former SNO detector was a Cherenkov detector, optimized for the detection of UV photons.

The bucket source measurement used a cylindrical UV transparent acrylic flask, the so-called bucket, housing about 11 of liquid scintillator. It was deployed into the water inside the AV. The investigated scintillator is raw LAB with $2 \mathrm{~g} / \mathrm{l}$ PPO.

The number $N_{\text {hit }}$ of PMTs firing in an event is used as a measure of $P H$. This number is corrected, accounting for the number of non-working PMTs and the number of PMTs that were hit more than once during one event. One event refers to a trigger window of $400 \mathrm{~ns}$. The $P H$ scale is calibrated using an americium-beryllium (AmBe) source.

\subsection{Calibration}

To determine the light output of electrons, an AmBe source was attached to the bucket, producing neutrons and $4.4 \mathrm{MeV}$ $\gamma$-rays in ${ }^{9} \mathrm{Be}(\alpha, n \gamma){ }^{12} \mathrm{C}$ reactions. After thermalization, neutrons are mostly captured on ${ }^{1} \mathrm{H}$, producing a deuteron and a $2.2 \mathrm{MeV} \gamma$-ray. The two distinct Compton edges of known electron energy, shown in Fig. 9, enable the calibration of the light output scale, using Eq. 3 with $P H=N_{\text {hit }}$. Analogous to the calibration described in Sect. 2.2, each Compton edge is located in the $N_{\text {hit }}$ spectrum by a fit including the effect of the detector resolution and by inspecting the underlying distribution without the impact of the resolution. The resulting best fit calibration parameters of Eq. 3 are $m=(489 \pm 3) / \mathrm{MeV}$ and $a=-28.6 \pm 8.5$.

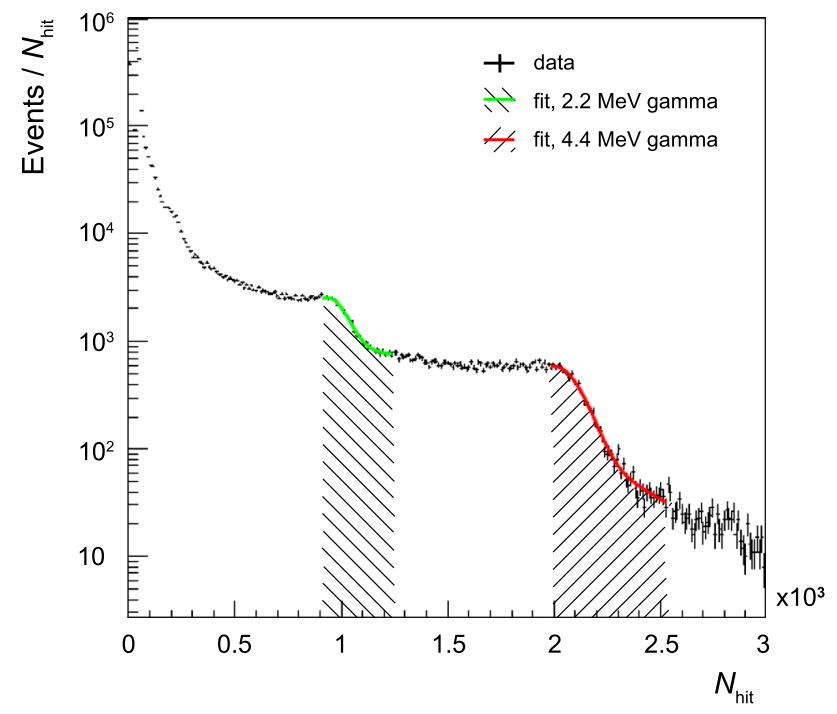

Fig. $9 N_{\text {hit }}$ distribution of an AmBe calibration source measured with $\mathrm{LAB}+2 \mathrm{~g} / \mathrm{l}$ PPO. Also shown are fits to the Compton edges resulting from the direct $4.4 \mathrm{MeV} \gamma$-rays and the delayed $2.2 \mathrm{MeV} \gamma$-rays from neutron capture on ${ }^{1} \mathrm{H}$ 


\subsection{Determination of the $\alpha$-particle response}

Internal $\alpha$-particles were produced by dissolved ${ }^{222} \mathrm{Rn}$ gas in the scintillator. ${ }^{222} \mathrm{Rn}$ itself emits with a branching ratio of $99.9 \% \alpha$-particles with an energy of $5.49 \mathrm{MeV}$, producing ${ }^{218}$ Po which also decays under the emission of an $\alpha$ particle. The ${ }^{218}$ Po $\alpha$-particle has an energy of $6.00 \mathrm{MeV}$ and the branching ratio for this decay is $99.98 \%$. The third kind of $\alpha$-particles identified within the bucket source data has an energy of 7.69 MeV and results from decaying ${ }^{214} \mathrm{Po}$, a daughter nuclide further down the natural ${ }^{238} \mathrm{U}$ decay chain. This decay mode is the only one of ${ }^{214} \mathrm{Po}$.

${ }^{214}$ Po has a very short half-life $T_{1 / 2}$ of about $164.3 \mu \mathrm{s}$. ${ }^{214}$ Po events are thus easily identified making use of their time coincidence with the $\beta$-decay of the parent isotope, ${ }^{214} \mathrm{Bi}$ (see also [12]). To locate the ${ }^{218} \mathrm{Po}$ and ${ }^{222} \mathrm{Rn}$ lines, the background, which is dominated by $\beta$-particles and $\gamma$ rays from ${ }^{214} \mathrm{~Pb}$ and ${ }^{214} \mathrm{Bi}$ decays, is first subtracted. The shape of the corresponding background sum spectrum is determined via simulation, which was performed with the SNO software package, SNOMAN [30], which was kindly made available by the SNO collaboration. Figure 10 shows 600000 simulated ${ }^{214} \mathrm{Bi}$ and ${ }^{214} \mathrm{~Pb}$ decays. For comparison, the approximate positions of the ${ }^{222} \mathrm{Rn},{ }^{218} \mathrm{Po}$ and ${ }^{214} \mathrm{Po} \alpha$ peaks are indicated by vertical lines. It is found that the background decreases smoothly in the region where the $\alpha$-peaks are expected. The shape is well-described by a 6 th order polynomial.

The bucket source data were taken in several runs. Figure 11 shows data from one run, as an example, before and

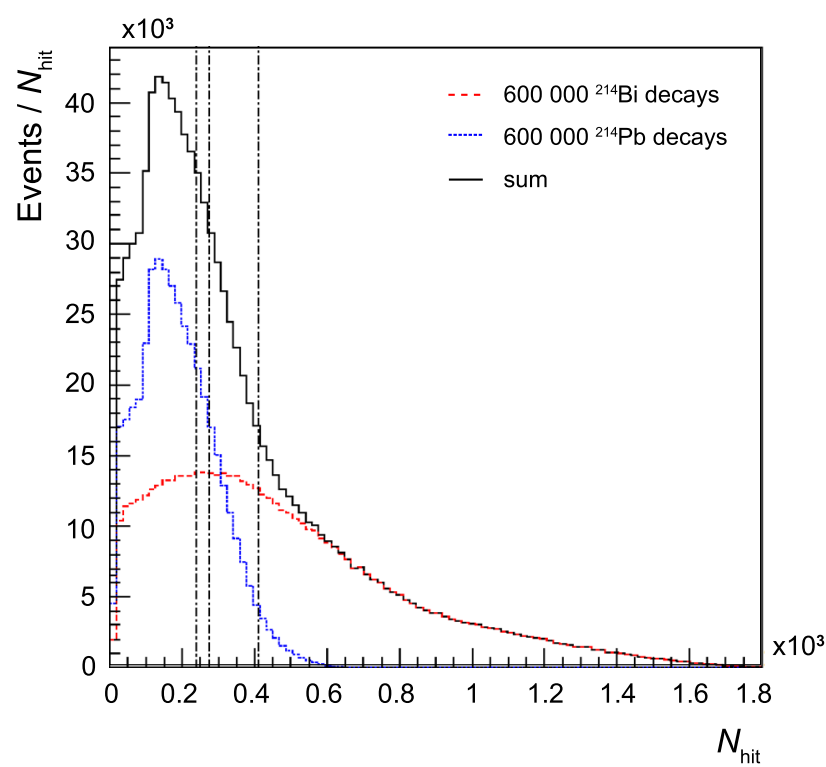

Fig. 10 Simulated $N_{\text {hit }}$ distributions of backgrounds from ${ }^{214} \mathrm{~Pb}$ and ${ }^{214} \mathrm{Bi}$ decays. The vertical dashed-dotted lines, in order of increasing $N_{\text {hit }}$ value, show the approximate positions of ${ }^{222} \mathrm{Rn},{ }^{218} \mathrm{Po}$ and ${ }^{214} \mathrm{Po}$ $\alpha$-peaks, respectively

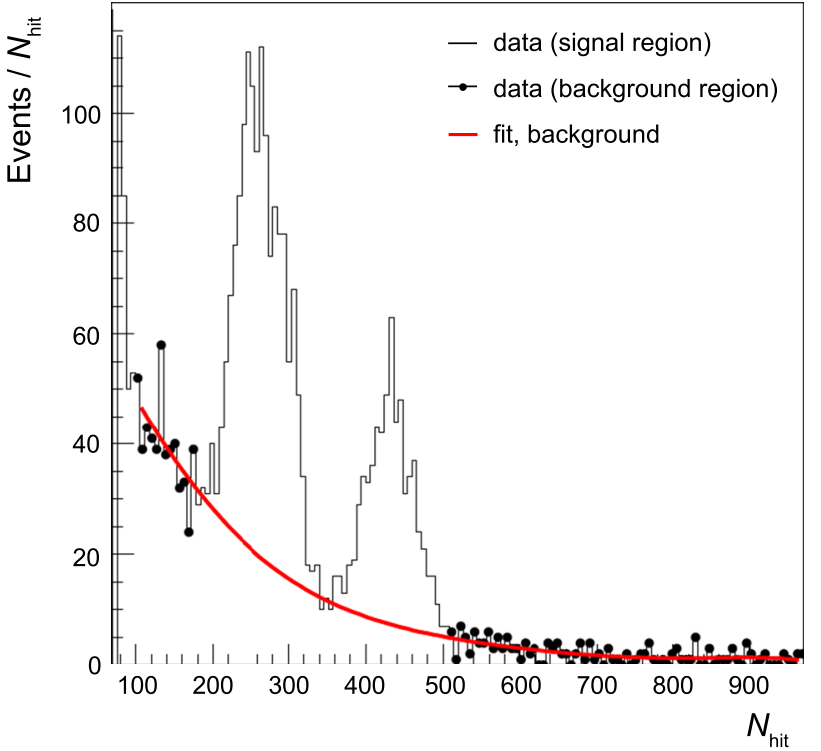

(a) Before background subtraction

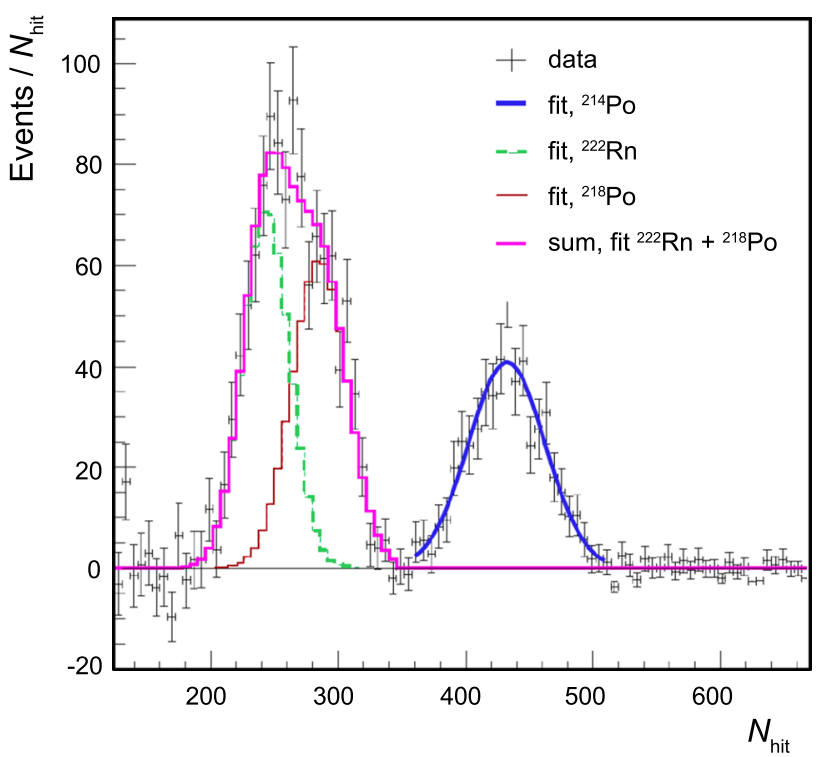

(b) After background subtraction

Fig. 11 Exemplary $N_{\text {hit }}$ distribution measured during one of the bucket source data taking runs. The fit of a 6 th order polynomial to the natural background, mainly from ${ }^{214} \mathrm{~Pb}$ and ${ }^{214} \mathrm{Bi}$ decays, is shown in (a). This background is subtracted in (b), where three Gaussian distributions are fitted to the $\alpha$-peaks from ${ }^{222} \mathrm{Rn},{ }^{218} \mathrm{Po}$ and ${ }^{214} \mathrm{Po}$

after background subtraction in the region of interest around the $\alpha$-peaks. Furthermore, in Fig. 11b the first peak is fitted with two Gaussian components, corresponding to ${ }^{222} \mathrm{Rn}$ and ${ }^{218}$ Po. The respective normalizations are constrained to be equal, assuming equilibrium. The means and standard deviations, instead, are allowed to float. The second peak, which is visible at a higher $N_{\text {hit }}$ value, is fitted with a single Gaussian function, accounting for the ${ }^{214}$ Po line with all parameters floating freely. The best fit normalization was found to be consistent with the equilibrium assumption. 
Table 1 Mean $N_{\text {hit }}$ values measured at an $\alpha$-particle energy $E_{\alpha}$ and light output $L$ in units of electron-equivalent energy after calibration. The uncertainty on $L$ results from error propagation of the uncertainties on $N_{\text {hit }}$ and the calibration parameters $m$ and $a$. The scintillator used is $\mathrm{LAB}+2 \mathrm{~g} / \mathrm{PPO}$

\begin{tabular}{llll}
\hline$\alpha$-Emitter & $E_{\alpha}[\mathrm{MeV}]$ & $N_{\text {hit }}$ & $L[\mathrm{MeV}]$ \\
\hline${ }^{222} \mathrm{Rn}$ & 5.49 & $246.4 \pm 2.2$ & $0.56 \pm 0.02$ \\
${ }^{218} \mathrm{Po}$ & 6.00 & $284.2 \pm 2.1$ & $0.64 \pm 0.02$ \\
${ }^{214} \mathrm{Po}$ & 7.69 & $422.2 \pm 2.6$ & $0.92 \pm 0.02$ \\
\hline
\end{tabular}

The best fit values of the Gaussian mean and the respective uncertainties provide the three data points listed in Table 1. Also listed are the values of the resulting light output $L$ in units electron-equivalent energy. The uncertainty of $L$ is obtained by propagating the uncertainties of the calibration parameters and the observed $N_{\text {hit }}$ value. A fit of Birks' law Eq. 1 to the three values of $L$ yields $k B=(0.0072 \pm$ $0.0003) \mathrm{cm} / \mathrm{MeV}$. Also in this analysis, $(\mathrm{d} E / \mathrm{d} x)(E)$ is calculated using SRIM. The best fit function is presented together with the bucket source data points in the next section.

\section{Comparison of the results from the three independent $\alpha$-particle quenching experiments}

All light output data points, measured with the three presented experiments, are shown in Fig. 12. For better visibility, the results from the two LAB samples used for the neutron beam experiment are compared to the other two experiments individually in Fig. 12a, b. Additionally shown are the individual best fit light output functions, parameterized by Birks' law Eq. 1. The corresponding best fit values are summarized in Table 2.

The best fit to the ${ }^{147} \mathrm{Sm}$ data reveals an overly small reduced $\chi^{2}$ value due to the fact that only a single data point exists which additionally has a large uncertainty. Furthermore, the obtained best fit $k B$ value has the largest uncertainty and is smaller than the results from the neutron beam and bucket source experiments. The large uncertainty on $k B$ results mainly from the uncertainty on the calibration parameters. It could be reduced in a future experiment by the use of an additional fluor, like bis-MSB, to improve the light output resolution and by the use of a greater variety of calibration sources with at best well-separated Compton edges. It is important to note, though, that all best fit $k B$ values, determined in the three different experiments, agree well within their total uncertainties.

The neutron beam data, observed with $\mathrm{LAB}+3 \mathrm{~g} / \mathrm{PPO}+$ $15 \mathrm{mg} / \mathrm{l}$ bis-MSB, is in excellent agreement with the bucket source data (see Fig. 12b) and also the fitted light output functions agree remarkably well. A slight tension among

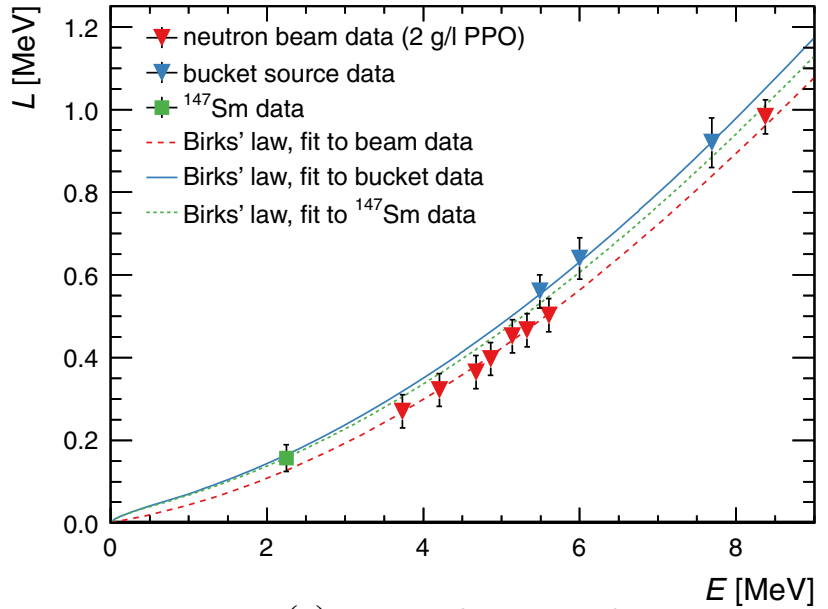

(a) Neutron beam sample: $2 \mathrm{~g} / \mathrm{lPPO}+15 \mathrm{mg} / \mathrm{l}$ bis-MSB

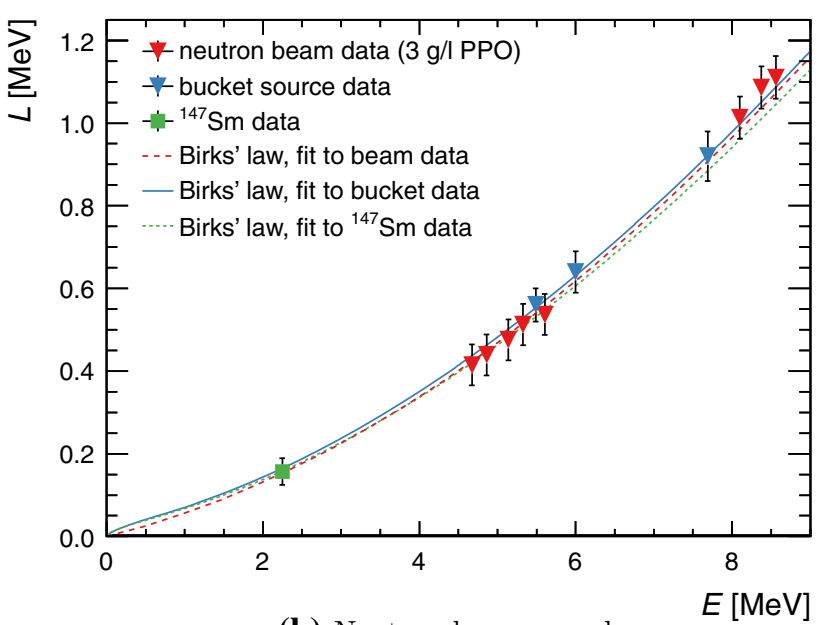

(b) Neutron beam sample: $3 \mathrm{~g} / \mathrm{lPPO}+15 \mathrm{mg} / \mathrm{l} \mathrm{bis}-\mathrm{MSB}$

Fig. 12 Light output $L$ of $\alpha$-particles in units of electron-equivalent energy as a function of the kinetic energy $E$. The LAB scintillator used for the data taken with the neutron beam contained $2 \mathrm{~g} / \mathrm{PPO}+15 \mathrm{mg} / \mathrm{l}$ bis-MSB (a) or $3 \mathrm{~g} / \mathrm{l} \mathrm{PPO}+15 \mathrm{mg} / \mathrm{l}$ bis-MSB (b). For an easier comparison, the same data from the bucket source and the samarium experiment is shown in both panels. The LAB scintillators used for these experiments contained $2 \mathrm{~g} / \mathrm{l} \mathrm{PPO}$ as the only fluor. Also shown is a fit of Birks' law Eq. 1 to each set of data points

data is observed in Fig. 12a though, where LAB + $2 \mathrm{~g} / \mathrm{lPPO}$ $+15 \mathrm{mg} / \mathrm{lbis}$-MSB was used in the neutron beam experiment. The neutron beam measurement is dominated by systematic uncertainties [19], where the systematic effects are mostly correlated. The correlations have been taken care of by including all sources of systematics as nuisance parameters in the $\chi^{2}$ fit, as discussed in [19]. Inspecting the individual pulls of the systematics revealed that the main contribution to the $\chi^{2}$ is due to the prompt $\gamma$-peak centroid position in the TOF spectrum and the slight non-linearity of the time-toamplitude converter (TAC) used for the TOF measurement. Thus the small tension among data is due to a systematic 
Table 2 Quenching parameter $k B$ for $\alpha$-particles in LAB based scintillator determined in three independent experiments. Also given in the table are the total uncertainties on $k B$ as well as the $\chi^{2}$ values over the number of degrees of freedom(ndf). The experiments are described in the text

\begin{tabular}{lll}
\hline Experiment (fluors) & $k B[\mathrm{~cm} / \mathrm{MeV}]$ & $\chi^{2} / \mathrm{ndf}$ \\
\hline $\begin{array}{l}\text { Neutron beam } \\
(2 \mathrm{~g} / \mathrm{l} \text { PPO }, 15 \mathrm{mg} / \mathrm{l} \text { bis-MSB) }\end{array}$ & $0.0076 \pm 0.0003$ & 0.73 \\
$\begin{array}{l}\text { Neutron beam } \\
(3 \mathrm{~g} / \mathrm{l} \text { PPO }, 15 \mathrm{mg} / \mathrm{l} \text { bis-MSB) }\end{array}$ & $0.0071 \pm 0.0003$ & 1.36 \\
$\begin{array}{l}\text { Bucket source } \\
(2 \mathrm{~g} / \mathrm{l} \mathrm{PPO})\end{array}$ & $0.0072 \pm 0.0003$ & 0.02 \\
$\begin{array}{l}\text { Samarium } \\
(2 \mathrm{~g} / \mathrm{l} \mathrm{PPO})\end{array}$ & $0.0066 \pm 0.0016$ & $2 \times 10^{-9}$ \\
\hline
\end{tabular}

uncertainty in the TOF measurement. It should be noted, though, that the corresponding neutron beam data obtained with $\mathrm{LAB}+2 \mathrm{~g} / \mathrm{l} \mathrm{PPO}+15 \mathrm{mg} / \mathrm{l}$ bis-MSB still agrees with the bucket source data within their standard measurement uncertainties. Also the corresponding best fit $k B$ values of $(0.0076 \pm 0.0003)$ and $(0.0072 \pm 0.0003) \mathrm{cm} / \mathrm{MeV}$, and thus the resulting functions $L(E)$, do not significantly deviate from each other.

\section{Comparison of the results from the simultaneous $\alpha$-particle and proton quenching measurements}

The $\alpha$-particle (see Sect. 2) and proton [19] quenching measurements using a neutron beam provide ideal conditions to test the hypothesis of a unique quenching parameter $k B$ for different ions in the same liquid scintillator. This hypothesis is subject to most present investigations [9] and important for all liquid scintillator particle detectors. The $\alpha$-particle quenching data was taken with the same detector and data acquisition system and was analyzed using the same method and assumptions as the proton quenching data discussed in [19]. Furthermore, the data was taken simultaneously and thus with the same scintillator filling. Hence, neither temperature changes, nor a different amount of impurities like oxygen, nor different aging could explain differences observed for different particle types. Finally, protons and $\alpha$-particles are both created inside the scintillator volume, thus avoiding surface effects.

The results for both particle types and scintillator samples are compared in Table 3 . While the presented results all agree for the same particle type measured with different scintillators based on the same solvent, the $k B$ values for protons ${ }^{7}$ and $\alpha$-particles in the same sample deviate by more than $5 \sigma$.

\footnotetext{
${ }^{7}$ It should be noted that [19] considers a quadratic correction term within Birks' law, parameterized by $C$. $C$ was found to be consistent with zero. It was verified for the present article that neglecting the correction term does not change the best fit $k B$ values.
}

Table 3 Quenching parameter $k B$ for protons and $\alpha$-particles in LAB based scintillator resulting from simultaneous neutron beam measurements. Also given are the total uncertainties. The proton quenching results are taken from [19]

\begin{tabular}{ll}
\hline Particle & $k B[\mathrm{~cm} / \mathrm{MeV}]$ \\
\hline $\mathrm{LAB}+2 \mathrm{~g} / \mathrm{l} \mathrm{PPO}+15 \mathrm{mg} / \mathrm{l}$ bis-MSB & \\
Proton & $0.0097 \pm 0.0002$ \\
$\alpha$-particle & $0.0076 \pm 0.0003$ \\
$\mathrm{LAB}+3 \mathrm{~g} / \mathrm{lPPO}+15 \mathrm{mg} / \mathrm{l} \mathrm{bis-MSB}$ & \\
Proton & $0.0098 \pm 0.0003$ \\
$\alpha$-particle & $0.0071 \pm 0.0003$ \\
\hline
\end{tabular}

The light output of the two particle types cannot be described by the same Birks parameter in the presented neutron beam measurements.

\section{Summary and outlook}

This article presents the results of $\alpha$-particle quenching measurements obtained with three independent experiments using LAB based liquid scintillators: the neutron beam experiment, the samarium experiment and the bucket source experiment. These experiments all use small liquid scintillator volumes, but apply different techniques. All three detectors have an increased sensitivity to UV light and thus Cherenkov light, which is additionally emitted when fast electrons traverse the scintillator. Nonetheless the wavelength sensitivities of the experiments are not identical. The $\alpha$-particles are internal in all presented measurements. The data analyses were performed under the same assumptions.

The energy dependent $\alpha$-particle light output is analytically described by Birks' law, which is parameterized by $k B$. The best fit value of $k B$ is determined by independent fits to two sets of neutron beam data points, to the bucket source data points and to the single ${ }^{147} \mathrm{Sm}$ data point. The four resulting $k B$ values range from $(0.0076 \pm 0.0003)$ to $(0.0066 \pm$ $0.0016) \mathrm{cm} / \mathrm{MeV}$. All presented measurements agree and the observed $\alpha$-particle responses can be described by the same $k B$ value within the uncertainties. The widest range of $\alpha$ particle energies is covered by the neutron beam experiment. It is thus recommended to use the parameter $k B$ resulting from this experiment. The value $(0.0076 \pm 0.0003) \mathrm{cm} / \mathrm{MeV}$, observed with $\mathrm{LAB}+2 \mathrm{~g} / \mathrm{lPPO}+15 \mathrm{mg} / \mathrm{l}$ bis-MSB, is slightly preferred over the one obtained with the LAB sample with $3 \mathrm{~g} / \mathrm{l} \mathrm{PPO}+15 \mathrm{mg} / \mathrm{l}$ bis-MSB . The determined reduced $\chi^{2}$ value in the first case is with 0.73 slightly lower than the one in the second case with 1.36 .

Within the neutron beam experiment, the proton light output was measured simultaneously with the $\alpha$-particle light output. The proton quenching analysis and results were previously presented in [19]. The $k B$ value obtained for protons is $(0.0097 \pm 0.0002) \mathrm{cm} / \mathrm{MeV}$ for $\mathrm{LAB}+2 \mathrm{~g} / \mathrm{lPO}$ 
$+15 \mathrm{mg} / \mathrm{l}$ bis-MSB and $(0.0098 \pm 0.0003) \mathrm{cm} / \mathrm{MeV}$ for $\mathrm{LAB}$ $+3 \mathrm{~g} / \mathrm{l} \mathrm{PPO}+15 \mathrm{mg} / \mathrm{l}$ bis-MSB. These proton $k B$ values deviate from the corresponding $\alpha$-particle $k B$ values by $6.4 \sigma$ and $5.8 \sigma$, respectively. Hence, the proton and the $\alpha$-particle light responses cannot be reproduced with the same $k B$ value. This observation is fundamental for current and future largescale liquid scintillator detectors, like $\mathrm{SNO}+$, which require a precise modeling of the signal and background light yield distribution over several $\mathrm{MeV}$ of electron-equivalent energy, induced by different kinds of particles. A reliable background model is crucial for e.g. the development of most efficient background rejection techniques.

This article demonstrates that the results obtained with the neutron beam experiment are transferable to the SNO+ experiment, despite non-identical wavelength sensitivities. This finding is very important, since it enables the transfer of also the proton quenching results [19] to the SNO+ MC model. It furthermore allows to examine further LAB based scintillators ex situ, which are of interest for $\mathrm{SNO}+$, like Teloaded LAB [12].

In future laboratory experiments, the influence of the wavelength sensitivity of the detector, particularly in the UV light region, should be systematically examined. This dependence is of interest for all liquid scintillator experiments in general. Besides this, the observed difference in the proton and $\alpha$-particle quenching parameters motivates systematic measurements using heavier ions under identical measurement conditions in order to investigate the dependence of $k B$ on ion properties like the mass. Though this investigation is of limited practical relevance for typical liquid scintillator experiments, it is of fundamental interest for the theory of liquid scintillation and the dependence of $k B$ on the ion type. This kind of measurement is only possible at accelerator facilities, where ion beams of sufficiently high energies can be produced to induce an observable amount of scintillation light.

Acknowledgments We acknowledge provision of the bucket source data by the SNO+ collaboration and software tools by the SNO collaboration. We further thank the mechanical workshop of the TU Dresden for the production of the scintillator cell as well as Kai Tittelmeier, the accelerator staff of the PTB and Andreas Hartmann from HZDR for their support. We give thanks to Arnd Sörensen for confirming the R2059-01 PMT gain stability. The LAB solvent was provided by Petresa Canada Inc., Bécancour QC. This work has been in part supported by the Deutsche Forschungsgemeinschaft (DFG), Germany (Grant no. ZU123/5), in part by the Science and Technology Facilities Council (STFC) of the United Kingdom (Grants No. ST/J001007/1, ST/K001329/1 and ST/M00001X/1) and in part by the U.S. Department of Energy under Contract No. DE-SC0012704.

Open Access This article is distributed under the terms of the Creative Commons Attribution 4.0 International License (http://creativecomm ons.org/licenses/by/4.0/), which permits unrestricted use, distribution, and reproduction in any medium, provided you give appropriate credit to the original author(s) and the source, provide a link to the Creative
Commons license, and indicate if changes were made. Funded by SCOAP ${ }^{3}$.

\section{References}

1. J.B. Birks, Proc. Phys. Soc. A 64, 874 (1951). doi:10.1088/ 0370-1298/64/10/303

2. J.B. Birks, The Theory and Practice of Scintillation Counting (Pergamon, New York, 1964)

3. E. Brannon, G.L. Olde, Radiat. Res. 16, 1 (1962)

4. C. Aberle, C. Buck, F.X. Hartmann, S. Schönert, S. Wagner, JINST 6, P11006 (2011). doi:10.1088/1748-0221/6/11/P11006

5. T. Novotný, PTB Report PTB-N-28 (1997)

6. H. Wan Chan Tseung, J. Kaspar, N. Tolich, Nucl. Instrum. Methods A 654, 318 (2011). doi:10.1016/j.nima.2011.06.095

7. T. Kögler et al., Nucl. Instrum. Methods A 701, 285 (2013). doi:10. 1016/j.nima.2012.10.059

8. T. Novotný, L. Büermann, S. Guldbakke, H. Klein, Nucl. Instrum. Methods A 400, 356 (1997). doi:10.1016/S0168-9002(97)00904-2

9. V.I. Tretyak, EPJ Web Conf. 65, 02002 (2014). doi:10.1051/ epjconf/20136502002, doi:10.1051/epjconf/20146502002

10. The Daya Bay Collaboration, Phys. Rev. Lett. 108, 171803 (2012). doi:10.1103/PhysRevLett.108.171803

11. The RENO Collaboration, Phys. Rev. Lett. 108, 191802 (2012). doi:10.1103/PhysRevLett.108.191802

12. The SNO+ Collaboration, Adv. High Energy Phys. 2016, 6194250 (2015). doi:10.1155/2016/6194250

13. L. Zhan, Nucl. Phys. Proc. Suppl. B 237-238, 114 (2013). doi:10. 1016/j.nuclphysbps.2013.04.071

14. S.-B. Kim, Nucl. Part. Phys. Proc. 265-266, 93-98 (2015). arXiv: 1412.2199

15. The LENS Collaboration, Phys. At. Nucl. 65, 2161 (2002). doi:10. $1134 / 1.1530294$

16. B. Cicenas, N. Solomey, Phys. Proced. 37, 1324 (2012). doi:10. 1016/j.phpro.2012.02.470

17. H.J. Brede et al., Nucl. Instrum. Methods 169, 349 (1980). doi:10. 1016/0029-554X(80)90928-3

18. H. Klein, H.J. Barrenscheen, G. Dietze, B.R.L. Siebert, W. Bretfeld, Nucl. Instrum. Methods 169, 359 (1980). doi:10.1016/ 0029-554X(80)90929-5

19. B. von Krosigk, L. Neumann, R. Nolte, S. Röttger, K. Zuber, Eur. Phys. J. C 73, 2390 (2013). doi:10.1140/epjc/s10052-013-2390-1

20. L.P. Ekström, R.B. Firestone, WWW Table of Radioactive Isotopes, database version 2/28/99. http://ie.lbl.gov/toi/. Accessed 22 April 2015

21. Petresa Canada Inc., Linear Alkylbenzene, Petrelab 500-Q (P 500Q) (2009). MSDS

22. G. Dietze, H. Klein, Nucl. Instrum. Methods 193, 549 (1982). doi:10.1016/0029-554X(82)90249-X

23. G. Dietze, H. Klein (1982). PTB Report PTB-ND-22

24. J.F. Ziegler, SRIM-2012 computer program. http://www.srim.org/. Accessed 16 Jan 2013

25. J.F. Ziegler, J. Appl. Phys. 85, 1249 (1999). doi:10.1063/1.369844

26. N.N. Greenwood, A. Earnshaw, Chemie der Elemente (VCH, Weinheim, 1988)

27. R. Beyera et al., Nucl. Instrum. Methods A 575, 449 (2007). doi:10. 1109/NSSMIC.2008.4774625

28. B.C. Arnold, R.J. Beaver, Test 11, 7 (2002)

29. A. Azzalini, The Skew-Normal and Related Families (Cambridge University Press, Cambridge, 2014). Part of Inst. of Math. Stat. Monographs

30. The SNO Collaboration, Nucl. Instrum. Methods A 449, 172 (2000). doi:10.1016/S0168-9002(99)01469-2 